\title{
Impact of the histone deacetylase inhibitor 4-phenylbutyrate on the clearance of apoptotic pancreatic carcinoma cells by human macrophages
}

\author{
LENA WELSCH $^{1 *}$, THILO WELSCH ${ }^{1 *}$, DMITRIY I. DOVZHANSKIY $^{1 *}$, KLAUS FELIX $^{1}$, \\ NATHALIA A. GIESE ${ }^{1}$, DMITRI V. KRYSKO ${ }^{2,3}$ and JENS WERNER ${ }^{1}$ \\ ${ }^{1}$ Department of General, Visceral and Transplantation Surgery, University of Heidelberg, Heidelberg, Germany; \\ ${ }^{2}$ Molecular Signaling and Cell Death Unit, Department for Molecular Biochemical Research, VIB and \\ ${ }^{3}$ Department of Biomedical Molecular Biology, Ghent University, Technologiepark 927, 9053 Ghent, Belgium
}

Received April 4, 2011; Accepted May 24, 2011

DOI: 10.3892/ijo.2011.1239

\begin{abstract}
Histone deacetylase inhibitors have been found to have potent anticancer activities, partly induced by tumour cell apoptosis. The clearance of apoptotic tumour cells is an important mechanism of antitumour immune surveillance. The aim of this study was to assess the impact of 4-phenylbutyrate (4-PB) and its immunological effects on the macrophage clearance of apoptotic pancreatic ductal adenocarcinoma (PDAC) cells. To this end, a co-culture system of human macrophages from donors and PDAC patients, and PDAC cell lines (T3M4, PANC-1 and AsPC-1) was established to study the effect of 4-PB. Apoptosis and phagocytic activity were analysed using flow cytometry, and phagocytosis was confirmed by confocal microscopy. Further, p21 expression was quantified by immunoblot analysis. 4-PB treatment $(0-10 \mathrm{mM})$ resulted in a dose-dependent induction of tumour cell apoptosis in two of the cell lines (T3M4 and PANC-1), but it also induced human macrophage apoptosis. The apoptotic effect of gemcitabine on PDAC cells was further enhanced by 4-PB. Moreover, 4-PB led to a dose-dependent overexpression of the cell cycle regulator p21 in tumour cells. In co-culture, apoptotic PDAC cells were phagocytosed by donor macrophages and phagocytosis was increased through tumour cell exposure to 4-PB and/or gemcitabine, whereas phagocytosis of PANC-1 cells was reduced using macrophages of PDAC patients treated with 4-PB. The 4-PB treatment induced human macrophage expression of the pro-angiogenic IL-8 and simultaneously inhibited inflammatory cytokine release through
\end{abstract}

Correspondence to: Dr Jens Werner, Department of General, Visceral and Transplantation Surgery, University of Heidelberg, Im Neuenheimer Feld 110, 69120 Heidelberg, Germany

E-mail: jens.werner@med.uni-heidelberg.de

${ }^{*}$ Contributed equally

Key words: 4-phenylbutyrate, histone deacetylase inhibitors, pancreatic cancer, apoptosis, phagocytosis, macrophages modulation of IL-10 and TNF $\alpha$ after phagocytosis of apoptotic PDAC cells. In conclusion, the 4-PB treatment activated tumour cell death in PDAC cells, resulting in tumour cell phagocytosis by macrophages. The latter were characterized by an anti-inflammatory and pro-angiogenic cytokine response demonstrating adverse, tumour-promoting effects of macrophages on tumour cells. Thus, the potential of 4-PB as an anticancer agent against PDAC cannot be reliably assessed without taking into account the complex tumour microenvironment.

\section{Introduction}

Currently, pancreatic ductal adenocarcinoma (PDAC) still has an extremely dismal prognosis. The best outcome is achieved through complete tumour resection and adjuvant chemotherapy with 5-fluorouracil (5-FU)- or gemcitabine-based regimens, with a median survival of about 23 months (1-3). Moreover, only $15-20 \%$ of patients who present with PDAC are potential candidates for curative resection. Gemcitabine is also the mainstay of palliative chemotherapy. Palliative treatment of locally advanced or metastatic PDAC results in a median survival of 6-7 months (4), reflecting the poor chemotherapeutic response of PDAC and the need for improved chemotherapeutic combinations.

Two important processes control the epigenetic modification of the DNA and histones: DNA-methylation and post-translational histone modification (5). An essential mechanism is the reversible acetylation and deacetylation of lysine residues of histones. These reactions are balanced and catalysed by histone acetyl transferases (HATs) and deacetylases (HDACs) (6). Histone acetylation is a key regulator controlling gene expression, for example tumour suppression genes, and dysregulation of these counteracting enzymes is coupled to the development of different malignancies $(7,8)$. Thus, HDAC deacetylase $\mathrm{N}$-terminal lysine groups act as co-repressor complexes to inhibit the transcription and condensation of chromatin, thereby influencing cell differentiation and cell cycles. Eighteen human HDAC have been identified and subdivided into classes I to IV based on their homology to yeast HDACs, their subcellular localization, or their enzymatic activities (9). 
Histone deacetylase inhibition leads to diverse antitumoural responses affecting cell differentiation, cell cycle arrest and apoptosis (9). According to their chemical structure, HDAC inhibitors (HDACi) are divided into six classes and currently about 10 to 15 HDACi are being studied in clinical trials $(10,11)$. Whereas mono-therapy with HDACi for haematological malignancies has yielded promising results, current concepts favour a combination treatment including HDACi for solid malignancies $(10,12)$. Up until now, only vorinostat was approved by the FDA for the mono-therapy of cutaneous T-cell lymphoma, but many other HDACi are being tested in phase II trials for treating both haematological and solid malignancies (13-15). 4-Phenylbutyrate (4-PB) was one of the first HDACi with a proven antitumour effect and was successfully used against acute promyelocytic leukaemia (16). Pharmacologic evaluation of 4-PB has underlined its tolerability, relative low toxicity, and high activity at micromolar serum concentrations (17).

A high expression of class I HDACs has recently been demonstrated in PDAC. Moreover, the selective inhibition of class I HDAC through valproic acid-induced TRAIL-mediated apoptosis in PDAC in vitro was observed (11). The treatment of PDAC cells in vitro by 4-PB consequently resulted in the induction of apoptosis, and the combination with gemcitabine had an overadditive effect on tumour cell apoptosis (18). Tumour growth, however, is controlled by the tumour microenvironment, which consists of different cell types including endothelial, stellate and host immune cells, as well as tumourassociated macrophages (TAMs). The role of TAMs within the tumour microenvironment and tumour-promoting signals is controversial and has been discussed (19), but no information is available about the activity of HDACi on the crosstalk between tumour cells and TAMs.

The present in vitro study was designed to assess the impact of 4-PB and its combination with gemcitabine on PDAC tumour cell apoptosis to analyse the phagocytosis of these apoptotic cells by macrophages from either human donors or PDAC patients, and to investigate the immunological response of macrophages towards these apoptotic cells.

\section{Materials and methods}

Preparation of mononuclear cells. Human blood samples were collected from healthy control people and from patients who underwent surgery for PDAC without neoadjuvant chemotherapy at the Department of General, Visceral and Transplantation Surgery, University of Heidelberg, in order to obtain peripheral blood mononuclear cells (PBMCs). Blood samples of PDAC patients $(n=5)$ were collected during tumour surgery and analysed separately. All participants of the study signed an informed consent according to the Declaration of Helsinki. The PBMCs were separated using Histopaque ${ }^{\circledR}-1077$ (Sigma-Alrich, Deisenhofen, Germany) according to the manufacturer's instruction.

Macrophage maturation was achieved as previously described (20). Briefly, after fluorescent labelling (see below) and seeding of the PBMCs at a concentration of 5x10 $/ 96$-well insert, the adherent monocytes were cultured for 6 days at $37^{\circ} \mathrm{C}$ with $5 \% \mathrm{CO}_{2}$ in freshly prepared medium (DMEM and F-12 at a 1:1 ratio, both PAA Laboratories $\mathrm{GmbH}$, Cölbe, Germany) supplemented with $1 \%$ antibiotics (penicillin/streptomycin) and without foetal bovine serum (FBS) during macrophage maturation (final concentration $5 \times 10^{4} /$ well).

Pancreatic cancer cell lines and cell culture conditions. Human pancreatic cancer cell lines (AsPC-1, PANC-1) were purchased from ATCC (Manassas, VA, USA), and T3M4 was a gift from R.S. Metzgar (Duke University, NC, USA). Cells were cultured at $37^{\circ} \mathrm{C}$ with $5 \% \mathrm{CO}_{2}$ in RPMI-1640 (PAA Laboratrories $\mathrm{GmbH}$ ) supplemented with $1 \%$ antibiotics (penicillin/streptomycin) and $10 \%$ foetal calf serum (FCS).

Induction and analysis of cell death by flow cytometry. 4-Phenylbutyrate (Tributyrate ${ }^{\circledR}$, EuroHealth, Mönsterås, Sweden) was diluted in phosphate-buffered saline (PBS) to a final concentration ranging from 0.5 to $10 \mathrm{mM}$, according to the indicated concentrations in each experiment. Gemcitabine (Synchem OHG, Felsberg, Altenburg, Germany) was applied at a final concentration of 0.01 and $0.05 \mathrm{mM}$ in PBS. All cells were treated and cultivated under the same conditions $\left(37^{\circ} \mathrm{C}\right.$ with $5 \% \mathrm{CO}_{2}$ in RPMI-1640) and were exposed to the drugs $24 \mathrm{~h}$ before the experiments.

The cell viability of macrophages and PDAC cells after exposure to 4-PB and/or gemcitabine was analysed using annexin V/ propidium iodide (PI)-staining (Annexin V-FITC Apoptosis Detection kit I, BD Biosciences, Heidelberg, Germany). This method allows a discrimination between early apoptotic (annexin V+/PI-) and late apoptotic as well as necrotic (annexin $\mathrm{V}^{+} / \mathrm{PI}^{+}$) and viable (annexin $\mathrm{V}^{-} / \mathrm{PI}^{-}$) cells. The staining was performed according to the manufacturer's instructions. Briefly, the cells were suspended in a buffer solution (10 mM Hepes/ $\mathrm{NaOH}$ at $\mathrm{pH} 7.4,140 \mathrm{mM} \mathrm{NaCl}, 2.5 \mathrm{mM} \mathrm{CaCl}_{2}$ ) at the final concentration of $1 \times 10^{6}$ cells $/ \mathrm{ml}$. After the addition of Annexin V-FITC, the cells were incubated for $15 \mathrm{~min}$ at room temperature without light exposure. Flow cytometry was performed using the FACS LSR II system (BD Biosciences). A total of 10,000 ungated events were acquired for each sample and the data were analysed with BD FACS Diva ${ }^{\circledR}$ with the CSD module (BD Biosciences). In order to determine late apoptotic cells, PI was added to the samples. Flow cytometry was performed immediately thereafter.

In vitro phagocytosis assay. For the phagocytosis analysis, $\mathrm{PDAC}$ cells were stained with a green fluorescent dye (CellTracker ${ }^{\mathrm{TM}}$ Green CMFDA, Invitrogen, Karlsruhe, Germany). The staining solution was prepared in accordance with the manufacturer's instructions. The carcinoma cells were then rinsed with PBS, supplemented with freshly prepared staining solution and incubated for $20 \mathrm{~min}$ at $37^{\circ} \mathrm{C}$ with $5 \% \mathrm{CO}_{2}$. After rinsing with PBS, the cells were put into a fresh culture medium. In contrast to the monocytes, the cells were stained shortly before starting the phagocytosis experiment. The monocytes were stained with a red fluorescent dye (CellTracker Orange CMRA, Invitrogen) before seeding and maturation over 6 days.

Apoptotic and labelled PDAC cells were then re-suspended in culture medium at a final concentration of $1 \times 10^{6} / \mathrm{ml} ; 100 \mu \mathrm{l}$ of this suspension was then co-incubated with macrophages $\left(5 \times 10^{4}\right.$, labelled with CellTracker Orange CMRA) for $2 \mathrm{~h}$. The co-incubation was prepared at $37^{\circ} \mathrm{C}$ with $5 \% \mathrm{CO}_{2}$ and stopped by gently re-suspending the cells. The resulting cell suspension contained macrophages and non-engulfed PDAC cells. Phagocytotic 
A

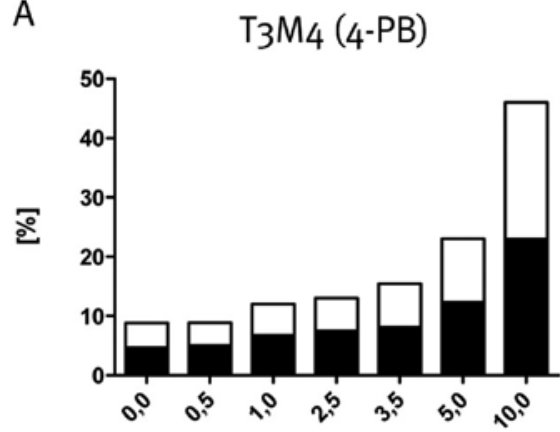

concentration [mM]

Early apoptosis

$\square$ Late apoptosis

C

PANC-1 (4-PB)

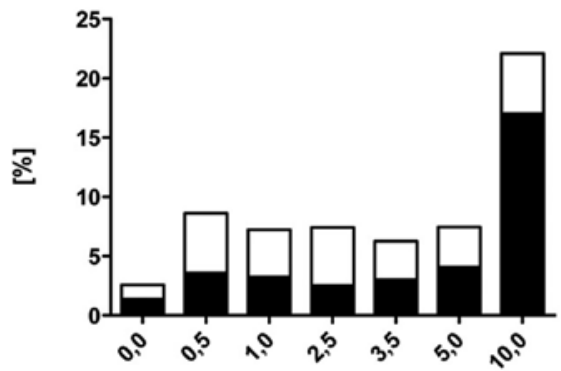

concentration [mM]

$\mathrm{E}$

AsPC-1 (4-PB)

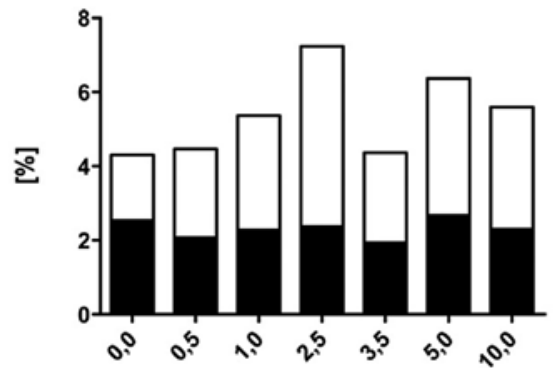

concentration [mM]
B

$\mathrm{T}_{3} \mathrm{M}_{4}$ (4-PB+gemcitabine)

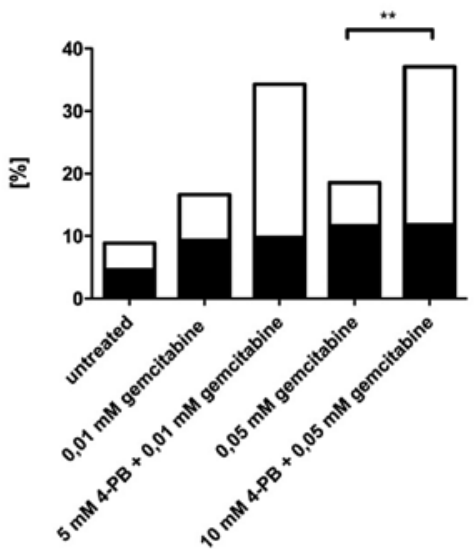

D PANC-1 (4-PB+gemcitabine)

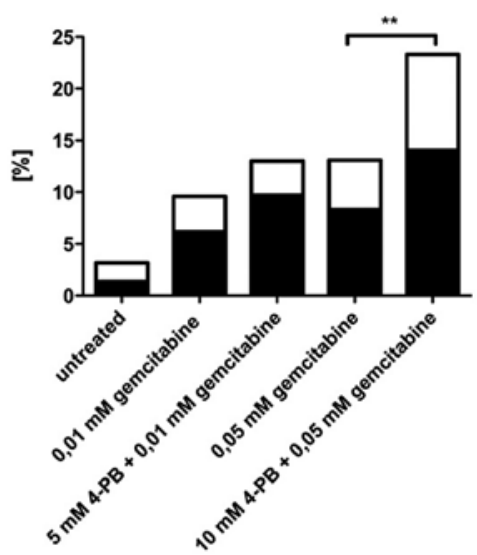

F AsPC-1 (4-PB+gemcitabine)

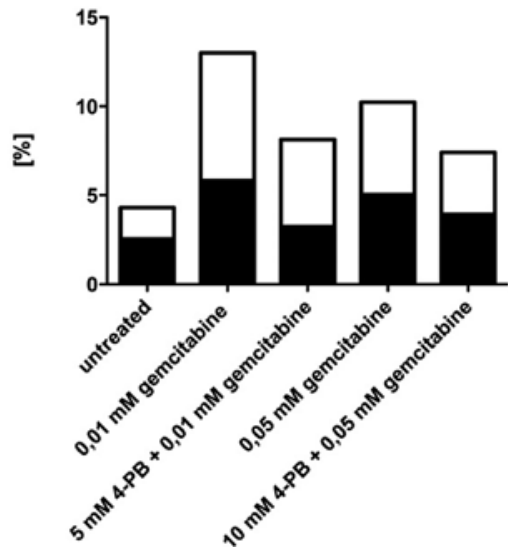

Figure 1. Early and late apoptosis in PDAC cell lines after treatment with 4-PB and gemcitabine. The percentage of apoptotic tumour cells after treatment with mono 4-PB ( $\mathrm{y}$-axis in $\mathrm{A}, \mathrm{C}, \mathrm{E})$ and 4-PB+gemcitabine $(\mathrm{B}, \mathrm{D}, \mathrm{F})$ stratified into early apoptotic and late apoptotic fractions in the different PDAC cell lines. In T3M4 (A and B), there was a dose-dependeny increase in apoptotic cells (early apoptosis, $\mathrm{P}=0.02$; late apoptosis, $\mathrm{P}=0.02$; Kruskal-Wallis), and in $\mathrm{PANC}-1$ (C and D) cells there was a significant increase in apoptosis from 5 to $10 \mathrm{mM} 4-\mathrm{PB}(\mathrm{P}<0.01)$. Moreover, the combination of 4-PB and gemcitabine significantly enhanced apoptosis in these cell lines $(\mathrm{P}<0.01)$. In contrast, the combination treatment did not lead to higher apoptosis in AsPC-1 (E and F) cells. The data are presented as mean values resulting from three independent experiments.

activity was determined by detecting the double fluorescence of the two dyes through flow cytometry. The supernatants of the original cell suspension were further used for cytokine expression experiments (see below). 
Confocal laser scanning microscopy. Phagocytosis was additionally assessed by confocal laser scanning microscopy. To this end, the co-cultured cells were seeded onto poly-L-lysine covered glass cover slips and fixed with $-20^{\circ} \mathrm{C}$ methanol. Confocal imaging was performed on a TCS-SP laser-scanning microscope using x40 or x63 oil-immersion objectives (Leica Microsystems, Heidelberg, Germany). Phagocytosis was confirmed if complete engulfment of tumour cells by macrophages was observed in the $\mathrm{x}, \mathrm{y}$ - and $\mathrm{x}, \mathrm{z}$-planes through the merged fluorescence.

Immunoblot. The cells were lysed in RIPA buffer. Protein samples (30 $\mu \mathrm{g}$ each) were heated to $99^{\circ} \mathrm{C}$ for $3 \mathrm{~min}$ and separated in a $4-12 \%$ gel (NuPAGE, Invitrogen, Carlsbad, CA). After blotting on a nitrocellulose membrane (Bio-Rad), the membranes were blocked in 5\% Slim Fast powder (Slim Fast, Allpharm Vertiebs-GmbH, Germany) diluted in TBS-buffer (Amresco, MoBiTec, Goettingen, Germany) at pH 7.5 with $0.05 \%$ Tween for $1 \mathrm{~h}$ at room temperature. The blots were then incubated with the primary antibody (rabbit anti-p21, 1:200, Abcam, Cambridge, UK; rabbit anti-GAPDH, 1:5.000, Cell Signaling) in blocking buffer overnight at $4^{\circ} \mathrm{C}$, rinsed for $1 \mathrm{~h}$ with washing buffer and incubated with horseradish peroxidase (HRP)-conjugated secondary antibodies for $1 \mathrm{~h}$ at room temperature. The blots were incubated in chemiluminescence solution (ECL; Amersham Biosciences, Freiburg, Germany) for 2 min and exposed to X-ray film. Before further primary antibody incubations, the membrane was exposed to stripping buffer (Thermo Scientific, Rockford, IL) for $45 \mathrm{~min}$ at $37^{\circ} \mathrm{C}$. Semiquantification of the immunoblot results was performed with the ImageJ software (National Institutes of Health, Bethesda, MD) based on triplicate experiments.

Cytokine analysis. Cytokine expression was measured in cell co-culture supernatants with a cytometric bead assay (BD Biosciences) by flow cytometry. The following cytokines were analysed: interleukin 8 (IL-8), IL-10 and tumour necrosis factor alpha $(\mathrm{TNF} \alpha)$. The samples were prepared according to the manufacturer's instructions. Briefly, the three cytokinespecific capture beads were incubated with detection buffer and the supernatant samples for $3 \mathrm{~h}$ at room temperature. The samples were then washed, centrifuged and re-suspended in washing buffer for flow cytometry (FACS LSR II system, BD, Heidelberg, Germany). The data were analysed using FCAP Array software (v.1.0.1, Soft Flow Hungary Ltd., Hungary).

Statistical analysis. Quantitative variables are expressed as means or as means \pm standard errors (SE) if not indicated otherwise. The Kruskal-Wallis test was used to analyse the correlation between apoptosis rates at different drug concentrations. Comparisons between the two groups were analysed by the Mann-Whitney U test (quantitative variables) or Student's t-test. Two-sided P-values were always computed and a difference was considered statistically significant at $\mathrm{P} \leq 0.05$. Graphs were plotted with GraphPad Prism 5.0 (GraphPad Software, Inc., La Jolla, CA).

\section{Results}

Apoptosis of tumour cells after 4-PB treatment. The investigation of apoptosis and cell death upon exposure to 4-PB and 4 -PB with gemcitabine demonstrated marked differences

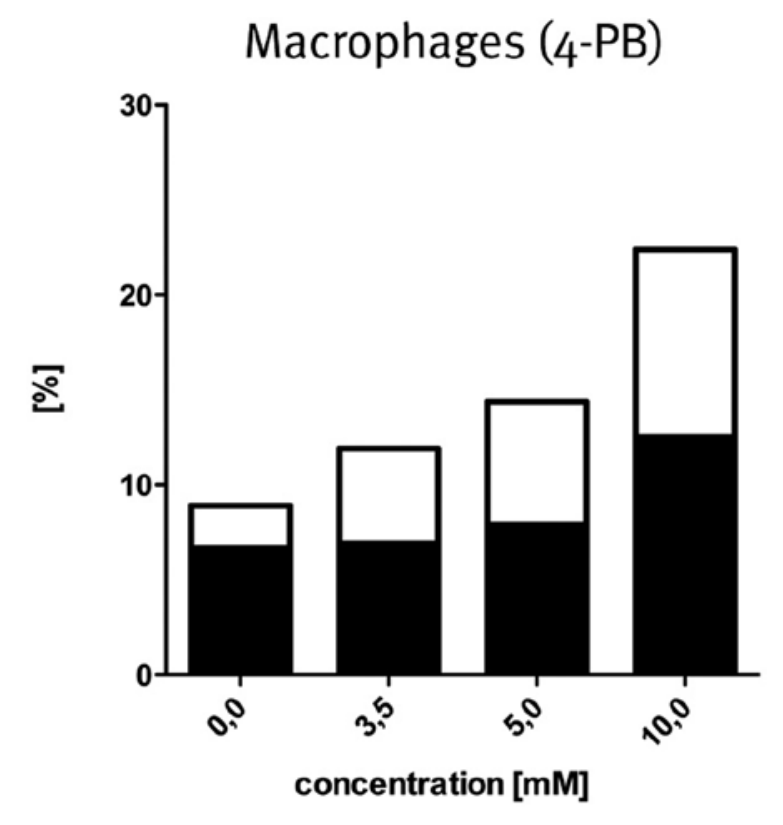

$\square$ Early apoptosis $\square$ Late apoptosis

Figure 2. Early and late apoptosis of healthy human donor macrophages after treatment with 4-PB. Healthy donor macrophages were treated with different doses of 4-PB over $24 \mathrm{~h}$ and apoptosis was assessed by flow cytometry. There was a significant increase in early and late apoptotic macrophages $(\mathrm{P}=0.015$, Kruskal-Wallis). The $y$-axis indicates the percentage of apoptotic cells of all analysed macrophages.

between the three PDAC cell lines. Early apoptosis was detected by annexin $\mathrm{V}$ positivity, whereas late apoptosis and cell death were detected by annexin $\mathrm{V}$ and PI positivity. A dose-dependent significant increase in early and late apoptotic tumour cells was observed in the T3M4 cell line after treatment with 0.5 to $10 \mathrm{mM}$ 4-PB (Fig. 1). The most significant difference in apoptotic cells occurred from 5 to $10 \mathrm{mM} 4-\mathrm{PB}(23.0 \pm 1.0 \%$ versus $46.0 \pm 7.3 \%$; $\mathrm{P}=0.04)$; likewise, 0.01 and $0.05 \mathrm{mM}$ gemcitabine both led to an increase in apoptosis. Furthermore, the combination of 4-PB and gemcitabine resulted in a significant increase of late apoptosis (Fig. 1B, P<0.01). In PANC-1 cells, 4-PB did not induce apoptosis in a clear dose-dependent manner; however, a strongly enhanced rate of apoptosis was noted at $10 \mathrm{mM} 4-\mathrm{PB}(7.5 \pm 2.0 \%$ at $5 \mathrm{mM} 4-\mathrm{PB}$ versus $22.1 \pm 3.2 \%$ at $10 \mathrm{mM} 4-\mathrm{PB}, \mathrm{P}=0.02$ ). In accordance with the T3M4 cells, the high concentration of 4-PB could significantly increase apoptosis in combination with gemcitabine in the PANC-1 cells (Fig. $1 \mathrm{C}$ and $\mathrm{D} ; \mathrm{P}<0.01$ ). In contrast, the AsPC-1 cell lines were not responsive to increasing doses of 4-PB regarding the induction of apoptosis (Fig. 1E) and only showed an insignificant increase in apoptosis after treatment with 4-PB and gemcitabine (Fig. 1F). The rate of apoptotic AsPC-1 cells was less than 7\% with 4-PB and less than $15 \%$ with 4-PB in combination with gemcitabine.

Apoptosis was also measured in mature human macrophages since the present study aimed to evaluate the effect of 4-PB not only on tumour cells, but also on the co-culture system of tumour cells and human mature macrophages. In vivo, both macrophages and tumour cells are exposed to drug concentrations if patients are treated with 4-PB. The rate of apoptotic macrophages increased in a dose-dependent manner following 
A

T3M4

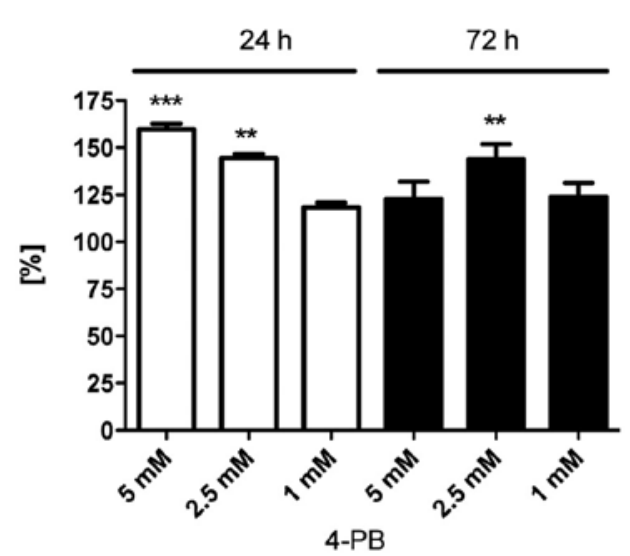

C

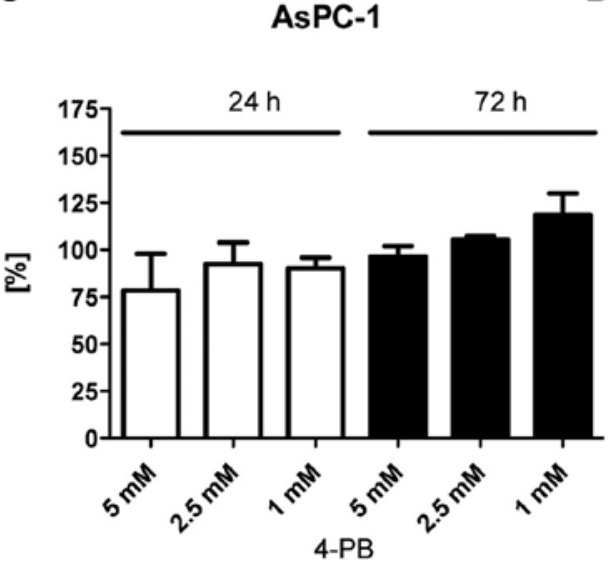

B

PANC-1

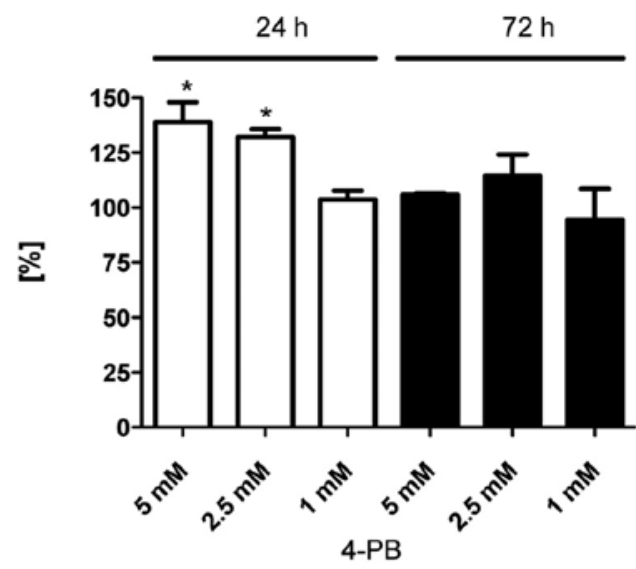

D

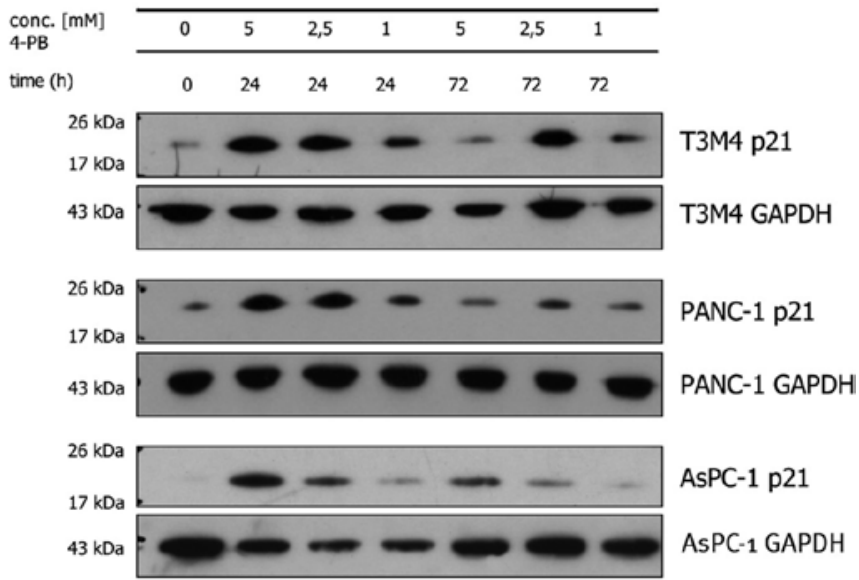

Figure 3. Expression of p21 after 4-PB treatment. (A-C) Quantification of p21 protein expression in T3M4, PANC-1 and AsPC-1 cells, respectively. The results are shown as relative expressions of p21 after 4-PB treatment in relation to the respective untreated controls. (D) Representative Western blot analyses of p21 expression in the cell lines T3M4, PANC-1 and AsPC-1. In summary, there was a significant increase in p21 expression after $24 \mathrm{~h}$ in T3M4 and PANC-1 cells, but not in AsPC-1 cells. Quantified data are based on independent triplicate measurements, ${ }^{*} \mathrm{P}<0.05,{ }^{* *} \mathrm{P}<0.01$.

4-PB treatment. At the final 4-PB concentration of $10 \mathrm{mM} 4-\mathrm{PB}$, $22.4 \pm 3.6 \%$ of the macrophages were apoptotic (Fig. 2).

Expression of $p 21$. It is currently unclear exactly how HDACi induce cell death pathways that lead to apoptosis, autophagy or necrosis $(21,22)$. Protein p21 is frequently found to be upregulated during cell cycle arrest and it appears to be required for apoptosis in a cell-type dependent manner (23). The elevation of p21 has been demonstrated in cancer cells after apoptotic stimuli and after HDACi treatment (24-26). Therefore, we investigated the expression of p21 in PDAC cells after treatment with 4-PB.

Increasing doses of 4-PB from 0 to $5 \mathrm{mM}$ over $24 \mathrm{~h}$ caused a significant upregulation of $\mathrm{p} 21$ expression in T3M4 and PANC-1 cells, whereas p21 expression peaked at $2.5 \mathrm{mM} 4-\mathrm{PB}$ when the drug treatment was prolonged over $72 \mathrm{~h}$ (Fig. 3A and B). Overall, 4-PB treatment of AsPC-1 cells did not reveal a significant impact on $\mathrm{p} 21$ expression, although single experiments were in line with the findings in the other cell lines, i.e. a 4-PB-dependent increase in p21 expression (Fig. 3C and D).

Phagocytosis of tumour cells by human macrophages. First, the phagocytosis of PDAC cells by human macrophages after treatment with 4-PB and/or gemcitabine was demonstrated and visualized by confocal microscopy. During the phagocytotic process, the macrophages completely engulfed the PDAC cells, which was visualized in the $\mathrm{x}, \mathrm{y}-$ and the $\mathrm{x}, \mathrm{z}-$ plane for T3M4 (Fig. 4A-F) and AsPC-1 cells (G-L). The double fluorescence signal resulting from phagocytosis was quantified by flow cytometry in further experiments.

Phagocytosis of PDAC cells by macrophages was significantly induced by pharmacologic treatment of the tumour cells in the three cell lines T3M4, PANC-1 and AsPC-1; however, the macrophages engulfed these three different cell lines with different efficiencies (Fig. 5A-C). In T3M4 and PANC-1 tumour cells, phagocytosis was significantly increased through treatment of the tumour cells with gemcitabine $(0.05 \mathrm{mM}), 4-\mathrm{PB}$ $(10 \mathrm{mM})$ and a combination of these two drugs, resulting in an increase in tumour cell apoptosis. However, the combination of gemcitabine and 4-PB did not lead to a significant enhancement of tumour cell phagocytosis compared to mono-therapy with gemcitabine, although the combination treatment increased tumour cell apoptosis. There was a tendency towards increased phagocytosis when the tumour cells had been treated with $0.05 \mathrm{mM}$ gemcitabine and the macrophages with $3.5 \mathrm{mM}$ 4-PB in T3M4 and PANC-1 cells. However, this was not significant (group 5). Interestingly, phagocytosis of PANC-1 cells (treated 


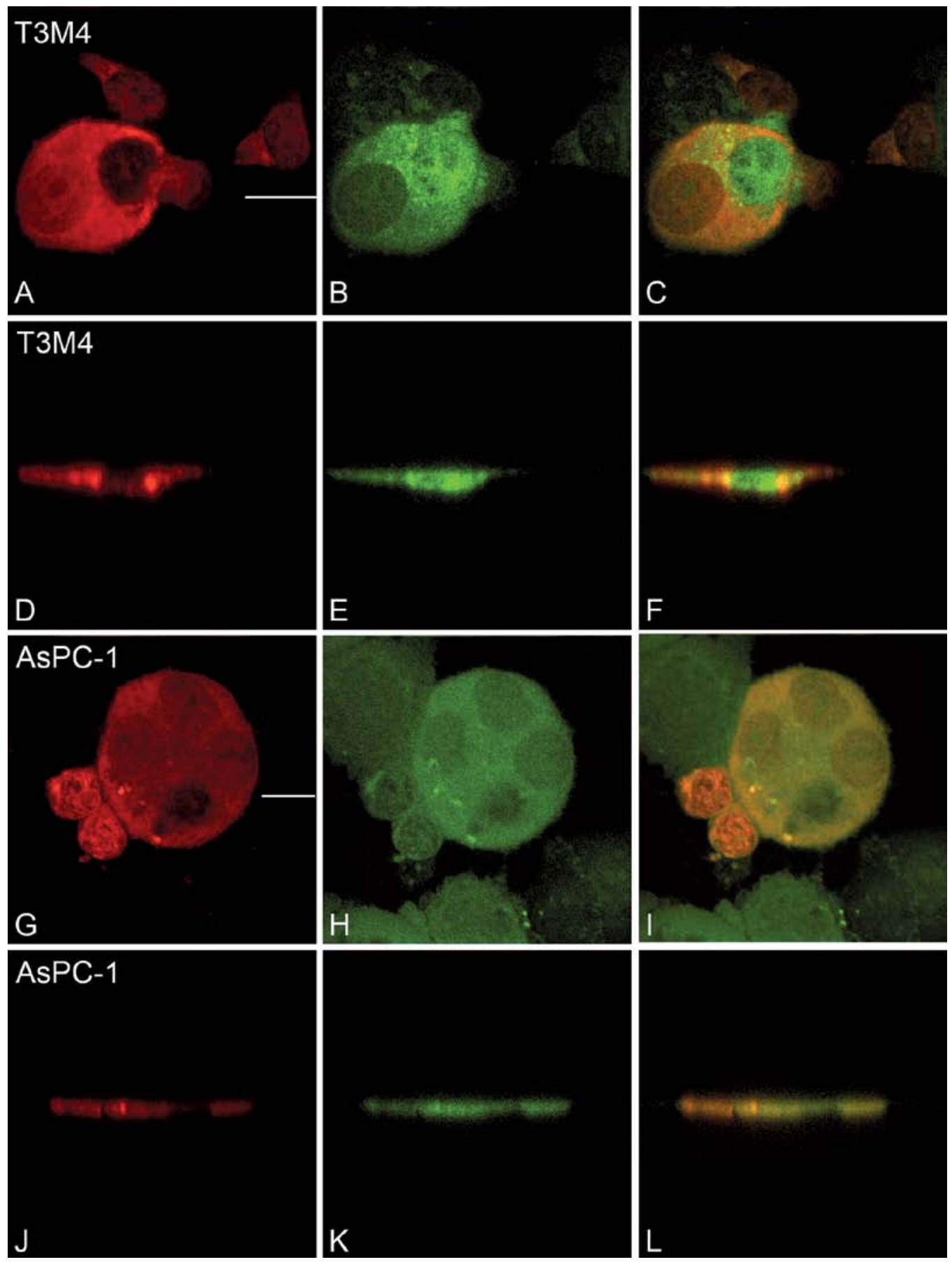

Figure 4. Confocal fluorescence imaging of phagocytosed tumour cells. (A-C) A macrophage (red) completely engulfed a T3M3 tumour cell during phagocytosis. (D-F) Complete engulfment of the tumour cell was confirmed by z-scan imaging. (G-I) Phagocytosis of AsPC-1 tumour cells. (J-L) Complete engulfment of an AsPC-1 tumour cell by a macrophage in the z-plane. Image widths represent $54 \mu \mathrm{m}$ each.

with $0.05 \mathrm{mM}$ gemcitabine) was significantly reduced when macrophages from PDAC patients were used and treated with $3.5 \mathrm{mM}$ 4-PB (Fig. 5B, compare groups 5 and 6). Similar, but non-significant results with a decreased phagocytosis rate by PDAC patient macrophages were found in T3M4 cells (Fig. 5A).

There was no such finding regarding an increased phagocytosis rate in PDAC patient macrophages or a reduction of phagocytosis if the patient macrophages were treated with 4-PB in AsPC-1 cells (Fig. 5C). The phagocytosis index using AsPC-1 cells did not exceed $17 \%$, on average.
Cytokine expression in macrophages after tumour cell phagocytosis. We further investigated the cytokine response of human macrophages to the phagocytosis of 4-PB- and control-treated T3M4 tumour cells. According to recent reports, phagocytosis of apoptotic tumour cells ignites an anti-inflammatory response, whereas necrotic cells trigger a pro-inflammatory response (27). We observed an increased IL-8 and IL-10 response in donor and PDAC patient macrophages after $2 \mathrm{~h}$ of tumour cell (T3M4, $10 \mathrm{mM} 4-\mathrm{PB}$ ) phagocytosis (Fig. 6A and B). On the contrary, the expression of TNF $\alpha$ was significantly decreased after phagocy- 
A

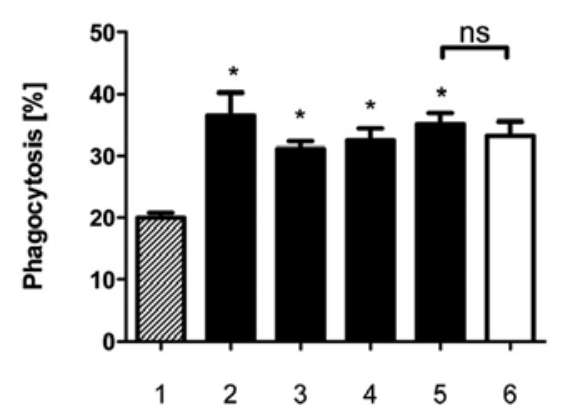

B

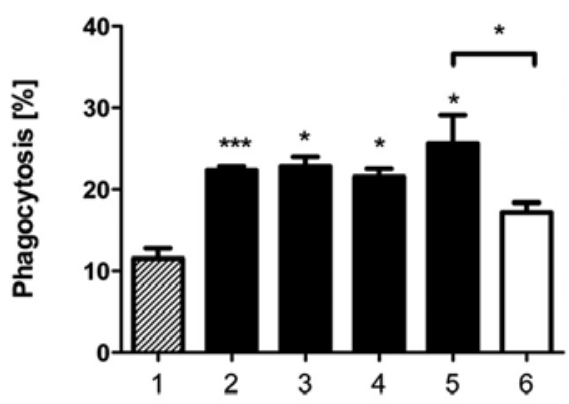

AsPC-1

C

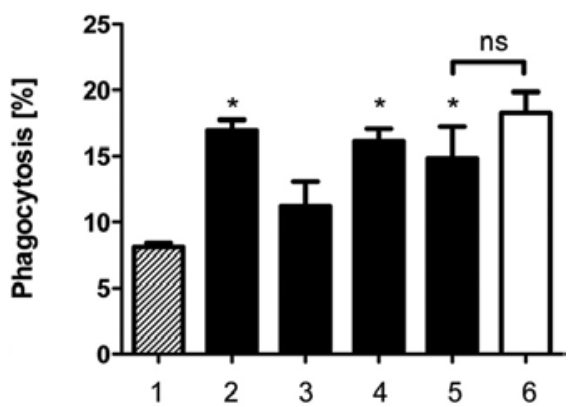

Figure 5. Tumour cell phagocytosis by tumour-associated macrophages after treatment with 4-PB and gemcitabine. Phagocytosis indices after phagocytosis and co-culture of T3M4 (A), PANC-1 (B), and AsPC-1 (C). The phagocytosis index was calculated as the number of phagocytosing macrophages divided by the number of detected macrophages, and represents the phagocytotic activity of the macrophages. The data are presented as the mean \pm SE resulting from five independent experiments. The numbers along the abscissa indicate the different treatment groups: 1, control; 2, PDAC cells treated with 0.05 mM gemcitabine; 3 , PDAC cells treated with $10 \mathrm{mM}$ 4-PB; 4, PDAC cells treated with $10 \mathrm{mM}$ 4-PB and $0.05 \mathrm{mM}$ gemcitabine; 5, PDAC cells treated with 0.05 mM gemcitabine and macrophages with $3.5 \mathrm{mM}$ 4-PB; 6, PDAC cells treated with $0.05 \mathrm{mM}$ gemcitabine and PDAC patient macrophages with $3.5 \mathrm{mM} 4-\mathrm{PB}$. $\mathrm{P}<0.05$; ${ }^{* *} \mathrm{P}<0.01$. Statistical differences are related to the control group results (1).

A

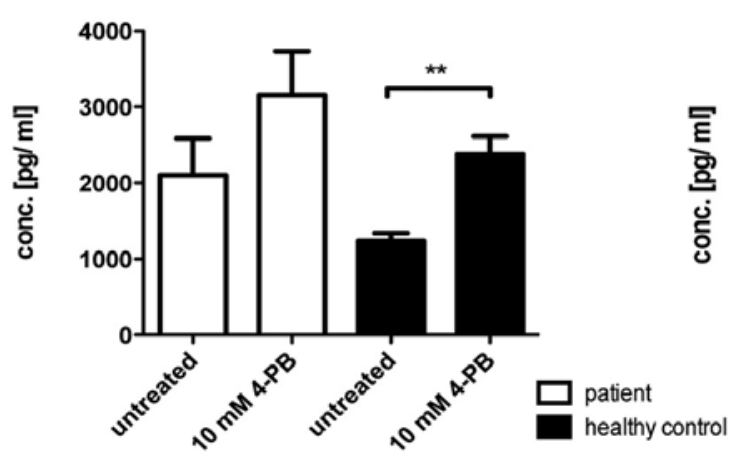

B

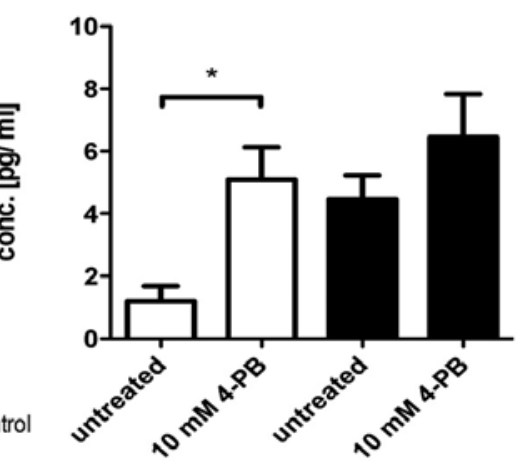

C

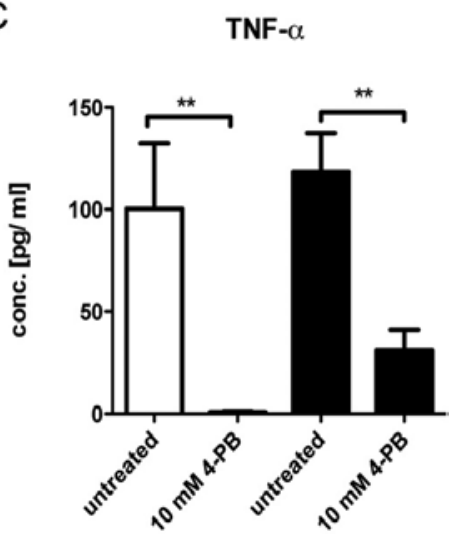

Figure 6. Cytokine expression after T3M4 tumour cell phagocytosis by macrophages (donor and PDAC patient macrophages). The expression of IL-8 (A), IL-10 (B) and TNF $\alpha(C)$ in the macrophages after phagocytosis of 4-PB-treated T3M4 cells compared to mock-treated T3M4 cells. The expression of interleukin (IL) 8 and 10, and of TNF $\alpha$ after phagocytosis. The tumour cells were treated with 4-PB or mock treated for $24 \mathrm{~h}$ and phagocytosis was allowed in a co-culture system for 2 h. ${ }^{*} \mathrm{P}<0.05 ;{ }^{* *} \mathrm{P}<0.01$

tosis of 4-PB-treated tumour cells compared to the phagocytosis of untreated tumour cells (Fig. 6C).

\section{Discussion}

Escape from apoptotic signalling pathways is an essential feature of the chemotherapeutic resistance of PDAC cells (28). Apoptotic tumour cells elicit responses in neighbouring cells of the tumour microenvironment. Such cellular responses include migration, proliferation, and differentiation as well as the production of immunomodulatory mediators and engulfment of the apoptotic cells by macrophages $(29,30)$. The present study was designed to test the HDACi 4-PB as an anticancer agent through activating apoptotic cell death in PDAC cells. This is relevant, because HDAC can inhibit the extrinsic TRAILinduced apoptosis in PDAC (11). On the other hand, apoptotic tumour cell phagocytosis by tumour-associated macrophages (TAMs) is known to evoke tumour-promoting, anti-inflamma- tory cytokine secretion $(29,31)$, and modern strategies focus on manipulating the immune response of phagocytes for developing anticancer therapies (27). It is because of this complex interplay between tumour cells and their microenvironment that we investigated not only the cytotoxic effect on tumour cells, but also the influence on the adoptive tumour cell clearance by host macrophages.

First, 4-PB induced apoptosis in the PDAC cell lines T3M4 and PANC-1. Our results did not allow for an exact discrimination of late apoptosis and cell death by necrosis, but early apoptotic events constantly paralleled late apoptosis events, supporting the theory that apoptosis is the critical cell death pathway observed. The induction of apoptosis was not seen in the AsPC-1 cell line. We did not further investigate the reason for this apoptosis resistance, but it has been shown that AsPC-1 cells are insensitive to some apoptotic signals of the phosphatidylinositol 3'-kinase pathway as well (32). In accordance with our results, Ammerpohl et al demonstrated the induction of 
apoptosis in T3M4 and PANC-1 cells by 4-PB and a synergistic, overadditive effect by treatment with 4-PB and gemcitabine (18). Despite these interesting and promising data advocating the use of 4-PB as a supplemental therapeutic agent for the treatment of PDAC, the effects of 4-PB on the tumour microenvironment were not elucidated in previous studies. It is not clear whether non-malignant cells like primary human fibroblasts, stellate cells or peripheral blood monocytes are less sensitive to the drug or not. Our results demonstrate that human macrophages were highly sensitive to 4-PB treatment and that up to $20 \%$ of the cells were in apoptotic states.

4-Phenylbutyrate-induced apoptosis in PDAC cells was further characterized by expression analysis of $\mathrm{p} 21$. The cellcycle regulator p21 was found to be upregulated in the early stages of PDAC carcinogenesis (23). Previous data suggest that p21 is an inhibitor of cell-cycle progression in some malignancies $(33,34)$. The dose-dependent upregulation of p21 in tumour cells in the present study are in line with a decreased tumour growth rate after 4-PB treatment (18). Likewise, overexpression of p21 was detected after treatment of bladder and prostate cancer with belinostat, another HDACi $(35,36)$. The present study shows an initial dose-dependent increase within the first $24 \mathrm{~h}$ followed by a delayed decrease of p21 upregulation after $24 \mathrm{~h}$. One possible explanation is a sustained protein degradation in the extracellular space in the surroundings of accumulating apoptotic and necrotic cells.

In addition to previous studies that tested the effect of 4-PB on tumour cells (18), the present study examined the impact on tumour cell phagocytosis as well as on the immune response of macrophages (TAM-model) to approximate the tumour microenvironment essential for tissue homeostasis and tumour control in vivo. Phagocytosis of PDAC cells was upregulated by chemotherapeutic treatment with 4-PB and gemcitabine. Macrophages isolated from PDAC patients showed a reduced rate of tumour cell phagocytosis when treated with 4-PB. This suggests that PDAC might also influence peripheral blood mononuclear cells and affect the TAM immune response.

Importantly, 4-PB treatment enhanced TAM secretion of the pro-angiogenic IL-8. It further attenuated inflammatory signals by stimulation of the anti-inflammatory IL-10 and inhibition of $\mathrm{TNF} \alpha$ secretion. Interleukin 8 (also known as CXCL8) conveys angiogenic signals in PDAC, probably by the activation of endothelial proliferation (37). High levels of the immunosuppressive cytokine IL-10 were found in PDAC, which helped the cancer cells to avoid immune recognition through the inhibition of immune cell activation (38-40). Interleukin 10 can also induce spontaneous apoptosis, thereby triggering a vicious cycle (41). In agreement, the suppression of TNF $\alpha$ further drives spontaneous apoptosis (41).

Together, the present results show that 4-PB induced PDAC cell apoptosis and that it also triggered anti-inflammatory and pro-angiogenic signals from activated macrophages after tumour cell phagocytosis that might exert an oncogenic drive. Consequently, adverse effects of 4-PB on the tumour microenvironment and the adaptive immune system have to be considered when testing 4-PB as therapeutic drug against PDAC. Further studies will have to be undertaken to determine whether or not anti-inflammatory and pro-angiogenic signals through 4-PB have relevant tumour-promoting effects on PDAC that limit the clinical use of 4-PB.

\section{Acknowledgements}

We thank Dieter Stephan for his excellent technical assistance for the flow cytometry. D.V.K. is a postdoctoral fellow paid by fellowships from FWO-Vlaanderen. The authors state no conflict of interest in this study.

\section{References}

1. Neoptolemos JP, Stocken DD, Bassi C, et al: Adjuvant chemotherapy with fluorouracil plus folinic acid vs gemcitabine following pancreatic cancer resection: a randomized controlled trial. JAMA 304: 1073-1081, 2010.

2. Neoptolemos JP, Stocken DD, Tudur Smith C, et al: Adjuvant 5-fluorouracil and folinic acid vs observation for pancreatic cancer: composite data from the ESPAC-1 and -3(v1) trials. Br J Cancer 100: 246-250, 2009.

3. Oettle H, Post S, Neuhaus P, et al: Adjuvant chemotherapy with gemcitabine vs observation in patients undergoing curative-intent resection of pancreatic cancer: a randomized controlled trial. JAMA 297: 267-277, 2007.

4. Cunningham D, Chau I, Stocken DD, et al: Phase III randomized comparison of gemcitabine versus gemcitabine plus capecitabine in patients with advanced pancreatic cancer. J Clin Oncol 27: 5513-5518, 2009.

5. Ho L and Crabtree GR: Chromatin remodelling during development. Nature 463: 474-484, 2010.

6. Marmorstein R: Protein modules that manipulate histone tails for chromatin regulation. Nat Rev 2: 422-432, 2001.

7. Gallinari P, Di Marco S, Jones P, Pallaoro M and Steinkuhler C: HDACs, histone deacetylation and gene transcription: from molecular biology to cancer therapeutics. Cell Res 17: 195-211, 2007.

8. Plumb JA, Finn PW, Williams RJ, et al: Pharmacodynamic response and inhibition of growth of human tumor xenografts by the novel histone deacetylase inhibitor PXD101. Mol Cancer Ther 2: 721-728, 2003.

9. Bolden JE, Peart MJ and Johnstone RW: Anticancer activities of histone deacetylase inhibitors. Nat Rev Drug Discov 5: 769-784, 2006.

10. Bots M and Johnstone RW: Rational combinations using HDAC inhibitors. Clin Cancer Res 15: 3970-3977, 2009.

11. Schuler S, Fritsche P, Diersch S, et al: HDAC2 attenuates TRAILinduced apoptosis of pancreatic cancer cells. Mol Cancer 9: 80, 2010.

12. Garcia-Manero G, Assouline S, Cortes J, et al: Phase 1 study of the oral isotype specific histone deacetylase inhibitor MGCD0103 in leukemia. Blood 112: 981-989, 2008.

13. Blum KA, Advani A, Fernandez L, et al: Phase II study of the histone deacetylase inhibitor MGCD0103 in patients with previously treated chronic lymphocytic leukaemia. Br J Haematol 147: 507-514, 2009.

14. Mackay HJ, Hirte H, Colgan T, et al: Phase II trial of the histone deacetylase inhibitor belinostat in women with platinum resistant epithelial ovarian cancer and micropapillary (LMP) ovarian tumours. Eur J Cancer 46: 1573-1579, 2010.

15. Tan J, Cang S, Ma Y, Petrillo RL and Liu D: Novel histone deacetylase inhibitors in clinical trials as anti-cancer agents. J Hematol Oncol 3: 5, 2010.

16. Warrell RP Jr, He LZ, Richon V, Calleja E and Pandolfi PP: Therapeutic targeting of transcription in acute promyelocytic leukemia by use of an inhibitor of histone deacetylase. J Natl Cancer Inst 90: 1621-1625, 1998.

17. Carducci MA, Gilbert J, Bowling MK, et al: A Phase I clinical and pharmacological evaluation of sodium phenylbutyrate on an 120-h infusion schedule. Clin Cancer Res 7: 3047-3055, 2001.

18. Ammerpohl O, Trauzold A, Schniewind B, et al: Complementary effects of HDAC inhibitor 4-PB on gap junction communication and cellular export mechanisms support restoration of chemosensitivity of PDAC cells. Br J Cancer 96: 73-81, 2007.

19. Lamagna $C$, Aurrand-Lions $M$ and Imhof BA: Dual role of macrophages in tumor growth and angiogenesis. J Leuk Biol 80: 705-713, 2006.

20. Bartel M, Hansch GM, Giese T, et al: Abnormal crosstalk between pancreatic acini and macrophages during the clearance of apoptotic cells in chronic pancreatitis. J Pathol 215: 195-203, 2008. 
21. Carew JS, Medina EC, Esquivel JA II, et al: Autophagy inhibition enhances vorinostat-induced apoptosis via ubiquitinated protein accumulation. J Cell Mod Med 14: 2448-2459, 2010.

22. Mottet $D$ and Castronovo V: Histone deacetylases: target enzymes for cancer therapy. Clin Exp Metastasis 25: 183-189, 2008.

23. Biankin AV, Kench JG, Morey AL, et al: Overexpression of $\mathrm{p} 21$ (WAF1/CIP1) is an early event in the development of pancreatic intraepithelial neoplasia. Cancer Res 61: 8830-8837, 2001.

24. Richon VM, Sandhoff TW, Rifkind RA and Marks PA: Histone deacetylase inhibitor selectively induces p21WAF1 expression and gene-associated histone acetylation. Proc Natl Acad Sci USA 97: 10014-10019, 2000.

25. Sheikh MS, Garcia M, Zhan Q, Liu Y and Fornace AJ Jr: Cell cycle-independent regulation of p21Waf1/Cip1 and retinoblastoma protein during okadaic acid-induced apoptosis is coupled with induction of Bax protein in human breast carcinoma cells. Cell Growth Differ 7: 1599-1607, 1996.

26. Sheikh MS, Li XS, Chen JC, Shao ZM, Ordonez JV and Fontana JA: Mechanisms of regulation of WAF1/Cip1 gene expression in human breast carcinoma: role of p53-dependent and independent signal transduction pathways. Oncogene 9: 3407-3415, 1994.

27. Krysko DV and Vandenabeele P: From regulation of dying cell engulfment to development of anti-cancer therapy. Cell Death Differ 15: 29-38, 2008.

28. Hamacher R, Schmid RM, Saur D and Schneider G: Apoptotic pathways in pancreatic ductal adenocarcinoma. Mol Cancer 7: 64, 2008

29. Gregory CD and Pound JD: Cell death in the neighbourhood: direct microenvironmental effects of apoptosis in normal and neoplastic tissues. J Pathol 223: 177-194, 2010.

30. Krysko DV and Vandenabeele P: Clearance of dead cells: mechanisms, immune responses and implication in the development of diseases. Apoptosis 15: 995-997, 2010.

31. Condeelis J and Pollard JW: Macrophages: obligate partners for tumor cell migration, invasion, and metastasis. Cell 124 263-266, 2006.
32. Bondar VM, Sweeney-Gotsch B, Andreeff M, Mills GB and McConkey DJ: Inhibition of the phosphatidylinositol 3'-kinaseAKT pathway induces apoptosis in pancreatic carcinoma cells in vitro and in vivo. Mol Cancer Ther 1: 989-997, 2002.

33. Migaldi M, Sgambato A, Garagnani L, et al: Loss of p21Waf1 expression is a strong predictor of reduced survival in primary superficial bladder cancers. Clin Cancer Res 6: 3131-3138, 2000

34. Natsugoe S, Nakashima S, Matsumoto M, et al: Expression of $\mathrm{p} 21 \mathrm{WAF} 1 / \mathrm{Cip} 1$ in the $\mathrm{p} 53$-dependent pathway is related to prognosis in patients with advanced esophageal carcinoma. Clin Cancer Res 5: 2445-2449, 1999.

35. Buckley MT, Yoon J, Yee H, et al: The histone deacetylase inhibitor belinostat (PXD101) suppresses bladder cancer cell growth in vitro and in vivo. J Transl Med 5: 49, 2007.

36. Qian X, Ara G, Mills E, LaRochelle WJ, Lichenstein HS and Jeffers M: Activity of the histone deacetylase inhibitor belinostat (PXD101) in preclinical models of prostate cancer. Int J Cancer 122: 1400-1410, 2008.

37. Matsuo Y, Ochi N, Sawai H, et al: CXCL8/IL-8 and CXCL12/ SDF-1alpha co-operatively promote invasiveness and angiogenesis in pancreatic cancer. Int J Cancer 124: 853-861, 2009.

38. Kim R, Emi M and Tanabe K: Cancer cell immune escape and tumor progression by exploitation of anti-inflammatory and proinflammatory responses. Cancer Biol Ther 4: 924-933, 2005.

39. Monti P, Leone BE, Zerbi A, et al: Tumor-derived MUC1 mucins interact with differentiating monocytes and induce IL-10highIL12low regulatory dendritic cell. J Immunol 172: 7341-7349, 2004.

40. Ohm JE and Carbone DP: VEGF as a mediator of tumor-associated immunodeficiency. Immunol Res 23: 263-272, 2001.

41. Ludewig B, Graf D, Gelderblom HR, Becker Y, Kroczek RA and Pauli G: Spontaneous apoptosis of dendritic cells is efficiently inhibited by TRAP (CD40-ligand) and TNF-alpha, but strongly enhanced by interleukin-10. Eur J Immunol 25: 1943-1950, 1995. 